\title{
Polynomial chaos representation of a stochastic preconditionner
}

\author{
Christophe Desceliers ${ }^{1}$, Roger Ghanem ${ }^{2},{ }^{*}$ and Christian Soize ${ }^{1}$ \\ ${ }^{1}$ Université Marne-La-Vallée, Paris, France ${ }^{2}$ The Johns Hopkins University, Baltimore, MD, USA
}

KEY WORDS: Stochastic systems; Preconditioners; Linear Solvers; Incomplete Factorization

\section{SUMMARY}

A method is developed in this paper to accelerate the convergence in computing the solution of stochastic algebraic systems of equations. The method is based on computing, via statistical sampling, a polynomial chaos decomposition of a stochastic preconditioner to the system of equations. This preconditioner can subsequently be used in conjunction with either chaos representations of the solution or with approaches based on Monte Carlo sampling. In addition to presenting the supporting theory, the paper also presents a convergence analysis and an example to demonstrate the significance of the proposed algorithm.

\section{Introduction}

In many problems of science and engineering the quest for accuracy in predicting the behavior of the associated physical systems has motivated the adoption of stochastic equations as viable representative models $[4,11,12]$. In many of these models, the governing equations take the form of partial differential equations with coefficients represented as stochastic processes or variables $[4,2]$. In discretizing these equations, linear algebraic systems ensue with entries that consist of generally correlated random variables $[4,10]$. A general and standard approach for estimating the solution of these stochastic equations is obtained through a Monte Carlo simulation logic that involves solving the full equations once for each realization in a statistical sample associated with the random system, and thus synthesizing a corresponding sample of the random solution [9]. The iterative solution of these equations would normally involve developing a different preconditioner for each of the samples. Consequently, if the number of samples is large and/or there are additional computational loops such as a frequency loop, a time loop, an equilibrium iteration loop for non linear problems, then the numerical effort associated with the construction of the preconditioners can become prohibitive. The main idea of this paper is to propose, as an initial step (outside the principal loops), the construction of an algebraic representation of a random preconditioner, called the stochastic preconditioner. Since the preconditioner is a random matrix which depends on the random parameters in the original

${ }^{*}$ Correspondence to: Roger Ghanem, 201 Latrobe Hall, Johns Hopkins University, baltimore, MD 21218 
matrix of the problem, this algebraic representation is chosen as the chaos decomposition of the random preconditioner. Once constructed, this preconditioner can then be used either in accelerating the convergence of Monte Carlo sampling or in producing computationally efficient estimates of the chaos decomposition of the solution [4].

The present paper deals with the problem where the linear algebraic system to be solved is frequency-dependent as would be the case in many dynamics problems. After describing the mathematical setting for the discretized problem in the next section, the stochastic preconditioners are introduced and a construction algorithm is described for their computation. An algorithm for estimating a solution of the stochastic equations is then presented followed by a convergence analysis. A numerical example is then used to expand on and demonstrate the various steps in building the preconditioner.

\section{Discretized equations in the frequency domain}

Consider the algebraic problem associated with solving the following linear system of equations,

$$
A(\omega, \xi) X(\omega)=F(\omega, \xi), \quad \omega \in B
$$

in which $\omega$ is a real parameter such as frequency, $\xi$ is an $R^{n}$-valued second-order random variable whose components are independent normalized Gaussian random variables, $A(\omega, \xi)$ is a random symmetric $(m \times m)$ complex matrix, $F(\omega, \xi)$ is a $C^{m}$-valued second-order random variable and $X(\omega)$ is a $C^{m}$-valued random variable. It is assumed that all rigid body components have been condensed-out, resulting in a matrix $A(\omega, \xi)$ that is invertible almost surely for every $\omega$. It is further assumed that random matrix $A(\omega, \xi)$ is such that, for every $\omega$ in $B$, vector $X(\omega)$ is a second-order random variable. blueIt is noted at this point that the randomness of $X(\omega)$ derives both from the randomness of operator $A(\omega, \xi)$ as well as from the randomness of the right hand side $F(\omega, \xi)$. The present thus covers situations where either one or both of these two quantities is stochastic. The functional dependence on $\omega$ is such that the treatment covers many frequency dependent problems where the analysis is conducted one frequency at a time. Similar dependence on other parameters is clearly covered by the present analysis.

Let $\left\{\Psi_{\alpha}(\xi)\right\}$ be the set of chaos variables in $\{\xi\}$,

$$
\Psi_{\alpha}(\xi): L^{2}\left(\Omega, R^{n}\right) \longrightarrow R, \quad \alpha=0,1, \ldots
$$

The variables form an orthogonal basis in the space $L^{2}$-space of second-order random variables and can be obtained as the multi-dimensional Hermite Polynomial functionals in a set of independent gaussian variables [4]. The chaos decomposition $X(\omega, \xi)$ of random variable $X(\omega)$ is of the form,

$$
X(\omega, \xi)=\sum_{\alpha} X_{\alpha}(\omega) \Psi_{\alpha}(\xi)
$$

in which $X_{\alpha}(\omega) \in C^{m}$ and such that

$$
X_{\alpha}(\omega)=\frac{\left\langle X(\omega, \xi) \Psi_{\alpha}(\xi)\right\rangle}{\left\langle\Psi_{\alpha}(\xi)^{2}\right\rangle} \quad \alpha=0,1, \ldots
$$

Prepared using nmeauth.cls

Int. J. Numer. Meth. Engng 20; :- 
Throughout this paper, for a multi-index $\alpha=\left(\alpha_{1}, \ldots, \alpha_{n}\right)$ of length $|\alpha|=\alpha_{1}+\ldots+\alpha_{n}$, each term in the sum $\sum_{|\alpha|=0}^{P}$ is itself the summation over all multi-indices of a given length.

The objective is to develop an acceleration procedure for the solution of Eq. (1) when either chaos developments or Monte Carlo Simulation (MCS) method is used in its solution $[4,5]$. In the case of the MCS method, a chaos decomposition is used. The proposed acceleration procedure relies on the development of stochastic preconditioners.

\section{Introducing stochastic preconditioners}

Let $C(\omega)$ be a frequency-dependent symmetric $m \times m$ complex random matrix. Introduce the change of variable,

$$
X(\omega)=C(\omega) Y(\omega)
$$

then Eq. (1) becomes

$$
A(\omega, \xi) C(\omega) Y(\omega)=F(\omega, \xi) .
$$

Since $A(\omega, \xi)$ is invertible a.s., the best stochastic preconditioner is clearly given by

$$
C(\omega)=A(\omega, \xi)^{-1},
$$

resulting in

$$
I Y(\omega)=F(\omega, \xi),
$$

where $I$ is the identity matrix which is deterministic. In this case, the solution $Y(\omega)$ associated with the ideal preconditioned stochastic equation (8) is easily obtained, either using chaos decomposition or using MCS method.

Equation (8) requires a knowledge of the stochastic inverse of $A(\omega, \xi)$ and is therefore an unrealistic limiting case. This paper proposes efficient stochastic preconditioners. It is noted here that in the general case, the preconditioner $C(\omega)$ will depend on the probabilistic parameters of the system and will therefore be denoted by $C(\omega, \xi)$.

For a given stochastic preconditioner $C(\omega, \xi)$, introduce the preconditioned stochastic matrix of the system

$$
A^{\text {cond }}(\omega, \xi)=A(\omega, \xi) C(\omega, \xi) .
$$

The stochastic solution to problem (1) is then obtained as

$$
\begin{array}{r}
X(\omega)=C(\omega, \xi) Y(\omega), \\
A^{\text {cond }}(\omega, \xi) Y(\omega)=F(\omega, \xi), \quad \omega \in B .
\end{array}
$$

There is a significant leeway in selecting $C(\omega, \xi)$. Certain properties are imposed on matrix $C(\omega, \xi)$ in order to maintain some desirable properties of the solution process, as well as the numerical behavior of the preconditioned system. These properties are as follows:

1. $X(\omega) \in L^{2}\left(\Omega, R^{m}\right), \quad \forall \omega \in B \quad$. 
2. $C(\omega, \xi)$ is a.s. invertible for every $\omega \in B$.

3. In general $C(\omega, \xi)$ is a full matrix. Consequently, $A^{\text {cond }}(\omega, \xi)$ is also full and such an approach would lead us to a prohibitive numerical effort for solving the resulting algebraic problem. A constraint on $C(\omega, \xi)$ should therefore be introduced to reduce this cost by maintaining a sparse representation of $A^{\text {cond }}(\omega, \xi)$.

\section{Construction of stochastic preconditioner}

Any approximation of $A(\omega, \xi)^{-1}$ satisfying the above properties is an acceptable preconditioner. From a design perspective, a compromise must be made between proximity to $A(\omega, \xi)^{-1}$ and the numerical effort associated with constructing the approximation. Two candidate preconditioners are analyzed below one of which is pursued in detail.

\subsection{Neumann series expansion}

Given the common usage of Neumann series approximations of the inverse operator in the context of stochastic computational mechanics, its significance as a preconditioner is worth highlighting. Indeed, the Neumann series expansion of $A(\omega, \xi)^{-1}$ provides a straightforward procedure for constructing a preconditioner. For a preconditioner constructed with an approximation of order $\mu$, the following expression is obtained,

$$
C(\omega, \xi)=\sum_{|\alpha|=0}^{\mu}(-1)^{\alpha}(A(\omega, \xi)-I)^{\alpha}
$$

where $I$ is the identity matrix. In general, for a given $A(\omega, \xi)$ the series is not convergent as $\mu \rightarrow \infty$ and the preconditioner defined by equation Eq. (11) using a finite sum could degrade the preconditioning of the original system instead of improving it. In addition, for $\mu \geq 2$, such a preconditioner is not a sparse matrix. Consequently, this preconditioner will not be pursued in this paper.

\subsection{Preconditioner based on the stochastic incomplete $L U$ factorization of the stochastic system matrix}

The construction of the stochastic incomplete LU factorization (see Refs. $[7,8,13,15,14,3]$ ) of stochastic system matrix $A(\omega, \xi)$ is an independent initial step in the construction of the stochastic solution of equation Eq. (1). Since for each $\omega \in B$, matrix $A(\omega, \xi)$ is invertible a.s., for each $\omega \in B$, it admits an incomplete factorization

$$
L^{\text {inc }}(\omega, \xi) U^{\text {inc }}(\omega, \xi)=L^{\text {inc }}(\omega, \xi) D^{\text {inc }}(\omega, \xi) L^{\text {inc }}(\omega, \xi)^{T} \quad, \quad \text { a.s. }
$$

In this factorization, $L^{\text {inc }}(\omega, \xi)$ is a random sparse triangular complex matrix having 1 on its diagonal and $D^{\text {inc }}(\omega, \xi)$ is a random diagonal complex matrix. Stochastic representations of $L^{\text {inc }}(\omega, \xi)$ and $D^{\text {inc }}(\omega, \xi)$ in terms of their respective chaos decompositions are given as 


$$
\begin{aligned}
L^{\mathrm{inc}}(\omega, \xi) & =\sum_{|\alpha|=0}^{P_{1}} L_{\alpha}^{\mathrm{inc}}(\omega) \Psi_{\alpha}(\xi), \\
D^{\mathrm{inc}}(\omega, \xi) & =\sum_{|\alpha|=0}^{P_{1}} D_{\alpha}^{\mathrm{inc}}(\omega) \Psi_{\alpha}(\xi),
\end{aligned}
$$

in which $L_{\alpha}^{\text {inc }}(\omega)$ and $D_{\alpha}^{\text {inc }}(\omega)$ are complex matrices with the same sparsity structure as $L^{\text {inc }}(\omega, \xi)$ and $D^{\text {inc }}(\omega, \xi)$ respectively, and are explicitly given by

$$
\begin{aligned}
L_{\alpha}^{\mathrm{inc}}(\omega) & =\frac{\left\langle L^{\mathrm{inc}}(\omega, \xi) \Psi_{\alpha}(\xi)\right\rangle}{\left\langle\Psi_{\alpha}(\xi)^{2}\right\rangle}, \\
D_{\alpha}^{\mathrm{inc}}(\omega) & =\frac{\left\langle D^{\mathrm{inc}}(\omega, \xi) \Psi_{\alpha}(\xi)\right\rangle}{\left\langle\Psi_{\alpha}(\xi)^{2}\right\rangle} .
\end{aligned}
$$

In the above expressions, the mathematical expectations are estimated as statistical averages from a finite sample population whose minimum size generally depends on $|\alpha|$. In the following, the size $N_{1}$ of this population will be taken as a function of $P_{1}$. The algorithm for computing $L_{\alpha}^{\text {inc }}$ and $D_{\alpha}^{\text {inc }}$ is then as follows. Starting with a sample $\xi^{(r)}$ of vector-valued random variable $\xi$, a corresponding sample $A\left(\omega, \xi^{(r)}\right)$ of random matrix $A(\omega, \xi)$ is synthesized. The corresponding sample of the incomplete factors $L^{\text {inc }}\left(\omega, \xi^{(r)}\right)$ and $D^{\text {inc }}\left(\omega, \xi^{(r)}\right)$ of matrix $A\left(\omega, \xi^{(r)}\right)$ is then computed. The approximations $L_{\alpha, N_{1}}^{\text {inc }}(\omega)$ and $D_{\alpha, N_{1}}^{\text {inc }}(\omega)$ of the coefficients $L_{\alpha}^{\text {inc }}(\omega)$ and $D_{\alpha}^{\text {inc }}(\omega)$ in the chaos decomposition of $L^{\text {inc }}(\omega, \xi)$ and $D^{\text {inc }}(\omega, \xi)$ are then computed from set $\left\{\xi^{(r)}\right\}$ by,

$$
\begin{aligned}
& L_{\alpha, N_{1}}^{\mathrm{inc}}(\omega)=\frac{1}{N_{1}\left\langle\Psi_{\alpha}(\xi)^{2}\right\rangle} \sum_{r=1}^{N_{1}} L^{\text {inc }}\left(\omega, \xi^{(r)}\right) \Psi_{\alpha}\left(\xi^{(r)}\right), \\
& D_{\alpha, N_{1}}^{\text {inc }}(\omega)=\frac{1}{N_{1}\left\langle\Psi_{\alpha}(\xi)^{2}\right\rangle} \sum_{r=1}^{N_{1}} D^{\text {inc }}\left(\omega, \xi^{(r)}\right) \Psi_{\alpha}\left(\xi^{(r)}\right) .
\end{aligned}
$$

Once the incomplete factorization of $A(\omega, \xi)$ has been computed as described above, the stochastic preconditioner can then be explicitly written as

$$
C(\omega, \xi)=\left(L^{\text {inc }}(\omega, \xi) D^{\text {inc }}(\omega, \xi) L^{\text {inc }}(\omega, \xi)^{T}\right)^{-1},
$$

in which $L^{\text {inc }}$ and $D^{\text {inc }}$ are given by Eq. (15). It should be noted that the stochastic preconditioner $C(\omega, \xi)$ is never constructed explicitly using Eq. (16) because the inversion of sparse matrices in that equation leads to a full matrix for $C(\omega, \xi)$. Indeed, since $L^{\text {inc }}$ is lower triangular, it is clear that $C(\omega, \xi)$ need not be computed explicitly since $L D L^{T}$ is easily solved by back substitution in each triangular system. An implicit procedure for implementing the preconditioning is next described.

\section{Solution of the stochastic system}

Refering to Section 2, the intermediate solution $Y(\omega, \xi)$ is first obtained as the solution to a conditioned system by using an iterative algorithm, followed by the evaluation of $X(\omega, \xi)$ 
using Eq. (10).

\subsection{Constructing the solution of the conditioned system}

For each sample $\xi^{(r)}$ of $\xi$ and using an iterative algorithm, the solution $Y^{(r)}(\omega)$ of the conditioned deterministic matrix equation

$$
A^{\text {cond }}\left(\omega, \xi^{(r)}\right) Y^{(r)}(\omega)=F\left(\omega, \xi^{(r)}\right),
$$

must be computed. As previously explained, sample $C\left(\omega, \xi^{(r)}\right)$ of stochastic preconditioner $C(\omega, \xi)$ is not explicitly computed. Rather, an iterative procedure involving $C^{-1}\left(\omega, \xi^{(r)}\right)$ is utilized for solving Eq. (17). In such a procedure, expressions of the form

$$
W=A^{\mathrm{cond}}\left(\omega, \xi^{(r)}\right) V
$$

have to be calculated. Using Eqs. (10) and (16), complex vector $W$ is given by

$$
W=A\left(\omega, \xi^{(r)}\right) R
$$

in which $R$ is the solution of the linear system

$$
C\left(\omega, \xi^{(r)}\right)^{-1} R=V
$$

which can be rewritten as

$$
L^{\mathrm{inc}}\left(\omega, \xi^{(r)}\right) D^{\mathrm{inc}}\left(\omega, \xi^{(r)}\right) L^{\mathrm{inc}}\left(\omega, \xi^{(r)}\right)^{T} R=V .
$$

The chaos decompositions of $L^{\text {inc }}(\omega, \xi)$ and $D^{\text {inc }}(\omega, \xi)$, given by Eq. (13) are utilized in Eq. (21). Given the lower triangular structure of $L^{\mathrm{inc}}\left(\omega, \xi^{(r)}\right)$, the solution of Eq. (21) is readily obtained using two back-substitutions. This process is very efficient due to the very sparse nature of $L^{\text {inc }}(\omega, \xi)$.

\subsection{Constructing a representation of stochastic solution $X(\omega, \xi)$}

A truncated decomposition of stochastic solution $X(\omega, \xi)$ is written as

$$
X_{P_{2}}(\omega, \xi)=\sum_{|\alpha|=0}^{P_{2}} X_{\alpha}(\omega) \Psi_{\alpha}(\xi)
$$

in which $X_{\alpha}(\omega)$ is given by

$$
X_{\alpha}(\omega)=\frac{\left\langle X(\omega, \xi) \Psi_{\alpha}(\xi)\right\rangle}{\left\langle\Psi_{\alpha}^{2}(\xi)\right\rangle} .
$$

Moreover, the first of Eqs. (10) is rewritten as

$$
C^{-1}(\omega, \xi) X(\omega, \xi)=Y(\omega, \xi)
$$

or, using Eq. (16),

$$
L^{\mathrm{inc}}(\omega, \xi) D^{\mathrm{inc}}(\omega, \xi) L^{\mathrm{inc}}(\omega, \xi)^{T} X(\omega, \xi)=Y(\omega, \xi)
$$

Prepared using nmeauth.cls 
An approximation $X_{\alpha, N_{2}}(\omega)$ of $X_{\alpha}(\omega)$ is evaluated using MCS method according to the expression

$$
X_{\alpha, N_{2}}(\omega)=\frac{1}{N_{2}\left\langle\Psi_{\alpha}^{2}(\xi)\right\rangle} \sum_{r=1}^{N_{2}} X\left(\omega, \xi^{(r)}\right) \Psi_{\alpha}\left(\xi^{(r)}\right),
$$

where $X\left(\omega, \xi^{(r)}\right)$ is obtained as the solution of Eq. (25) associated with sample $\xi^{(r)}$ of $\xi$. This solution is obtained mainly using two very sparse back-substitutions. Consequently, the approximation of $X_{P_{2}}(\omega, \xi)$ given by Eq. (22) is written as

$$
X_{P_{2}, N_{2}}(\omega, \xi)=\sum_{|\alpha|=0}^{P_{2}} X_{\alpha, N_{2}}(\omega) \Psi_{\alpha}(\xi) .
$$

\section{Convergence Analysis}

A general form of the chaos decomposition for a random quantity $Z(\omega, \xi)$ is given by

$$
Z_{P, N}(\omega, \xi)=\sum_{|\alpha|=0}^{P} Z_{\alpha, N}(\omega) \Psi_{\alpha}(\xi)
$$

in which

$$
Z_{\alpha, N}(\omega)=\frac{1}{N} \sum_{r=1}^{N} \frac{Z\left(\omega, \xi^{(r)}\right) \Psi_{\alpha}\left(\xi^{(r)}\right)}{\left\langle\Psi_{\alpha}^{2}(\xi)\right\rangle}
$$

Note that $P$ and $N$ are, in general, functions of $\omega$. blueThe value of $P$, required to achieve a certain level of accuracy, usually depends on the the departure of $Z(\omega, \xi)$ from a gaussian random variable, while the value of $N$ depends on the strength of the uncertainty as measured, for example, by its variance. A norm for the error estimation on these developments is given by

$$
\left|Z_{P, N}\right|^{2}=\left.\int_{B}||\left|Z_{P, N}(\omega)\right|\right|^{2} d \omega
$$

in which

$$
\begin{aligned}
\left.\left\|Z_{P, N}(\omega)\right\|\right|^{2} & =\left\langle\left\|Z_{P, N}(\omega, \xi)\right\|^{2}\right\rangle \\
& =\sum_{|\alpha|=0}^{P}\left\|Z_{\alpha, N}(\omega)\right\|^{2}\left\langle\Psi_{\alpha}^{2}(\xi)\right\rangle,
\end{aligned}
$$

and where $\|$.$\| is an appropriate norm. Note that P$ and $N$ are taken to be independent of $\omega$ in order to obtain a uniform convergence on $B$ with respect to $\omega$. This norm permits, given a specified level of tolerance, the calculation of the pair $\left(N^{\text {conv }}, P^{\text {conv }}\right)$ at convergence.

Prepared using nmeauth.cls 


\section{Numerical example}

\subsection{Definition of the mechanical system with random uncertainties}

Below, we consider a static problem which is then independent of the frequency parameter $\omega$. The stochastic system is a fixed structure consisting of an isotropic non-homogeneous linear elastic medium occupying a three-dimensional bounded domain $\Omega$ with boundary $\partial \Omega$, defined in Fig. 1. The structure is fixed on a part $\Gamma_{0}$ of $\partial \Omega$ for which the displacement field is zero (see Fig. 1). Domain $\Omega$ is the union of the three subdomains, $\Omega_{1}, \Omega_{2}$ and $\Omega_{3}$, as indicated in the figure. For $k=1,2,3$, subdomain $\Omega_{k}$ is occupied by a homogeneous medium whose Young modulus is $E_{k}$ and Poisson coefficient is $\nu_{k}$ such that $\nu_{k}=0.3$. The uncertainties concern only the Young moduli which are modeled by independent second-order random variables (i.e. of finite variance) whose mean values $\bar{E}_{k}=\left\langle E_{k}\right\rangle$ are such that $\bar{E}_{1}=2.1 \times 10^{10}, \bar{E}_{2}=2 \bar{E}_{1}$ and $\bar{E}_{3}=\bar{E}_{1} / 2$. For fixed $k$, random variable $E_{k}$ is written as

$$
E_{k}=\left(1-\frac{\delta_{k}}{\sqrt{2}}\right) \bar{E}_{k}+\frac{\delta_{k} \bar{E}_{k}}{\sqrt{2}} \xi_{k}^{2}
$$

In the above equation, $\xi_{k}$ is a normalized Gaussian random variable (centered with variance equal to 1 ) and $\delta_{k} \in[0, \sqrt{2}]$, is the coefficient of variation of $E_{k}$ which is defined by

$$
\delta_{k}=\sigma_{k} / \bar{E}_{k} \quad, \quad \sigma_{k}^{2}=\left\langle E_{k}^{2}\right\rangle-\bar{E}_{k}^{2} \quad,
$$

and permits the control of the dispersion of random variable $E_{k}$. It should be noted that the above construction results in $E_{k}>0$ almost surely. For the numerical calculations, the values of the dispersions parameters are $\delta_{1}=0, \delta_{2}=0.6$ and $\delta_{3}=0.8$ which means that subdomain $\Omega_{1}$ has no uncertainties (deterministic medium). The externally applied forces consist of 6 deterministic point forces (independent of $\xi$ ) denoted as $f_{1}, \ldots, f_{6}$, defined in Fig. 1 , the magnitudes of which are such that $\left|f_{2}\right|=\left|f_{5}\right|=\left|f_{6}\right|$ and $\left|f_{1}\right|=\left|f_{3}\right|=\left|f_{4}\right|=2\left|f_{2}\right|$.

The finite element mesh of the mechanical system is defined in Fig. 1 and is constituted of 8 -nodes isoparametric 3D solid finite elements. The number of DOFs is $m=1500$.

\subsection{Sparsity pattern of the random matrix of the system}

Let $X(\xi) \in R^{m}$ be the vector of the $m$ DOFs of the finite element model, such that

$$
A(\xi) X(\xi)=F,
$$

where $A(\xi)$ is the random stiffness matrix which is positive definite, $F$ is the vector of the external forces and $\xi=\left(\xi_{1}, \xi_{2}, \xi_{3}\right)$ is the random variable with values in $R^{3}$. In the following, the sparsity pattern $P_{A}$ of matrix $A(\xi)$ is defined as the set of all the non zero entries of random matrix $A(\xi)$. Taking into account Eq. (32) and since the random stiffness matrix depends linearly on random variables $E_{1}, E_{2}, E_{3}$, it can be deduced that random matrix $A(\xi)$ can be written as

$$
A(\xi)=A_{0}+A_{1} \xi_{1}^{2}+A_{2} \xi_{2}^{2}+A_{3} \xi_{3}^{2} \quad,
$$

in which the matrices $A_{0}, A_{1}, A_{2}$ and $A_{3}$ do not depend on $\xi$ (deterministic matrices) and have the same dimensions as $A(\xi)$. Consequently, sparsity pattern $P_{A}$ of random matrix $A(\xi)$ has an upper bound that is independent of $\xi$ and which is given by the sparsity pattern as $A_{0}$. Figure 2 displays sparsity pattern $P_{A}$ of matrix $A(\xi)$ or of matrix $A_{0}$. 
For any sample $\xi^{(r)}$ of random vector $\xi$, the complete factorization of $A\left(\xi^{(r)}\right)$ is written as $A\left(\xi^{(r)}\right)=L\left(\xi^{(r)}\right) D\left(\xi^{(r)}\right) L\left(\xi^{(r)}\right)^{T}$ in which $D\left(\xi^{(r)}\right)$ is a diagonal matrix and where the lower triangular matrix $L\left(\xi^{(r)}\right)$ has a sparsity pattern $P_{c}$. On the other hand, for any sample $\xi^{(r)}$, the incomplete factorization of $A\left(\xi^{(r)}\right)$ is written as $L^{\text {inc }}\left(\xi^{(r)} ; \ell\right) D^{\text {inc }}\left(\xi^{(r)} ; \ell\right) L^{\text {inc }}\left(\xi^{(r)} ; \ell\right)^{T}$ in which $D^{\text {inc }}\left(\xi^{(r)} ; \ell\right)$ is a diagonal matrix and where the lower triangular matrix $L^{\text {inc }}\left(\xi^{(r)} ; \ell\right)$ has a given sparsity pattern $P_{\ell}$ with upper bound independent of $\xi^{(r)}$. Note that the arguments for $L^{\text {inc }}$ and $D^{\text {inc }}$ in this context refer to random variables $\xi$ and a measure of bandwidth, $\ell$, and are different from arguments in equation (12).

It should also be noted that this sparsity pattern $P_{\ell}$ cannot be arbitrarily given, but has to be constructed so that the incomplete factorization can be carried out effectively for any sample $\xi^{(r)}$ of random vector $\xi$. For that, the following algorithm, adapted to this particular need, has been constructed :

1. The data are: the value $\ell$ of the bandwith of $L^{\text {inc }}(\xi ; \ell)$, with $\ell$ taken independent of $\xi$ and the knowledge of the sparsity pattern $P_{A}$ of matrix $A(\xi)$.

2. Calculation of the symbolic complete Cholesky factorization of matrix $A(\xi)$ allowing the sparsity pattern $P_{c}$ of the lower triangular matrix to be constructed.

3. From sparsity pattern $P_{c}$, construction of a sparsity pattern $\widetilde{P}_{\ell}$ whose bandwidth is smaller than or equal to $\ell$.

4. Sparsity pattern $P_{\ell}$ is deduced from sparsity pattern $\widetilde{P}_{\ell}$ by cancelling entries in $\widetilde{P}_{\ell}$ with the algorithm proposed in Ref. [13]. This is an algorithm which allows the incomplete factorization to be performed with the given sparsity pattern $P_{\ell}$.

\subsection{Chaos decomposition of the stochastic preconditioner}

Let $L_{P_{1}^{\prime}, N_{1}^{\prime}}^{\text {inc }}(\xi ; \ell)$ and $D_{P_{1}, N_{1}}^{\text {inc }}(\xi ; \ell)$ be the chaos decompositions given by Eqs. (13) of random matrices $L^{\text {inc }}(\xi ; \ell)$ and $D^{\text {inc }}(\xi ; \ell)$ where $P_{1}^{\prime}$ and $P_{1}$ are the chaos orders and where $N_{1}^{\prime}$ and $N_{1}$ are the numbers of samples used in estimating $L^{\text {inc }}(\xi ; \ell)$ and $D^{\text {inc }}(\xi ; \ell)$ respectively, and such that

$$
\begin{aligned}
& L_{P_{1}^{\prime}, N_{1}^{\prime}}^{\mathrm{inc}}(\xi ; \ell)=\sum_{|\alpha|=0}^{P_{1}^{\prime}} L_{\alpha, N_{1}^{\prime}}^{\mathrm{inc}}(\ell) \Psi_{\alpha}(\xi), \\
& D_{P_{1}, N_{1}}^{\mathrm{inc}}(\xi ; \ell)=\sum_{|\alpha|=0}^{P_{1}} D_{\alpha, N_{1}}^{\mathrm{inc}}(\ell) \Psi_{\alpha}(\xi),
\end{aligned}
$$

in which Eqs. (15) are rewritten as

$$
\begin{aligned}
& L_{\alpha, N_{1}^{\prime}}^{\mathrm{inc}}(\ell)=\frac{1}{N_{1}^{\prime}\left\langle\Psi_{\alpha}(\xi)^{2}\right\rangle} \sum_{r=1}^{N_{1}^{\prime}} L^{\mathrm{inc}}\left(\xi^{(r)} ; \ell\right) \Psi_{\alpha}\left(\xi^{(r)}\right), \\
& D_{\alpha, N_{1}}^{\mathrm{inc}}(\ell)=\frac{1}{N_{1}\left\langle\Psi_{\alpha}(\xi)^{2}\right\rangle} \sum_{r=1}^{N_{1}} D^{\mathrm{inc}}\left(\xi^{(r)} ; \ell\right) \Psi_{\alpha}\left(\xi^{(r)}\right) .
\end{aligned}
$$

The approximation of the chaos decomposition of $X(\xi)$ is computed by using Eqs.(26) and (27) in which $P_{2}$ is the decomposition chaos order and $N_{2}$ is the number of samples for 
approximating the mathematical expectation allowing the chaos coefficients to be computed. In order to calculate these coefficients, the following equation

$$
A\left(\xi^{(r)}\right) X\left(\xi^{(r)}\right)=F
$$

has to be solved for each sample $\xi^{(r)}$. For the present numerical example, this linear matrix equation is solved by using a preconditioned conjugate gradient solver (see, for instance, Ref.

[1]) with the stochastic preconditioner. Two methods are compared.

(1) Stochastic preconditioner: For the first one, the stochastic preconditioner $\left\{L_{P_{1}^{\prime}, N_{1}^{\prime}}^{\text {inc }}\left(\xi^{(r)} ; \ell\right), D_{P_{1}, N_{1}}^{\text {inc }}\left(\xi^{(r)} ; \ell\right)\right\}$ is defined by Eqs. (36) to (39).

(2) Usual preconditioner: For the second one, for each sample $\xi^{(r)}$, the preconditioner $\left\{L^{\text {inc }}\left(\xi^{(r)} ; \ell\right), D^{\text {inc }}\left(\xi^{(r)} ; \ell\right)\right\}$ of matrix $A\left(\xi^{(r)}\right)$ is computed using the usual incomplete factorization $([8])$.

\subsection{Convergence analysis}

7.4.1. Convergence analysis of the stochastic preconditioner with respect to the chaosdecomposition orders. In order to study the convergence of the stochastic preconditioner chaos decomposition with respect to orders $P_{1}$ and $P_{1}^{\prime}$, the graphs of the mappings $P_{1} \mapsto$ $\left\|\left.|| D_{P_{1}, N_{1}}^{\text {inc }}(\xi ; \ell)||\right|^{2} /\right\|\left|D_{1, N_{1}}^{\text {inc }}(\xi ; \ell) \|\right|^{2}$ and $P_{1}^{\prime} \mapsto\left\||| L_{P_{1}^{\prime}, N_{1}^{\prime}}^{\text {inc }}(\xi ; \ell)\left|\left\|^{2} /\right\|\right| L_{1, N_{1}^{\prime}}^{\text {inc }}(\xi ; \ell) \mid\right\|^{2}$ are constructed for $N_{1}=N_{1}^{\prime}=8000$ and for different values of the bandwidth $\ell$. The norm $\|\mid\|$. $\|$ is such that \|\|$\cdot \|^{2}=\left\langle\|\cdot\|^{2}\right\rangle$ in which $\|\cdot\|$ is the Frobenius norm. Figures 3 and 4 display these graphs. It can be seen that for all $\ell \leq 100$, acceptable convergence is achieved with a chaos decomposition order 2 for $D^{\text {inc }}(\xi ; \ell)\left(P_{1}=2\right)$ and a chaos decomposition order of 0 for $L^{\text {inc }}(\xi ; \ell)\left(P_{1}^{\prime}=0\right)$. This remarkable fact that matrix $L^{\text {inc }}(\xi ; \ell)(\xi ; \ell)$ is almost independent of $\xi(\xi ; \ell)$ implies that only one sample of $L^{\text {inc }}(\xi ; \ell)$ is necessary to construct its chaos decomposition which is then identical to its mean.

blueAs indicated previously in the paper, values of $P_{1}$ and $P_{1}^{\prime}$ indicate the departure of the components of random matrices $D^{\text {inc }}$ and $L^{\text {inc }}$ from a gaussian random variable. Although the probabilistic measure of elements of these two matrices is quite complex and is not easily amenable to analysis, further investigation will be required in order to analyze the convergence behavior of the chaos representation of the stochastic preconditioners.

7.4.2. Convergence analysis of the stochastic solution with respect to the chaos-decomposition order. For studying the convergence analysis of $X_{P_{2}, N_{2}}(\xi)$ with respect to the chaos decomposition order $P_{2}$, the graph of the mapping $\left.P_{2} \mapsto\left\|\left|X_{P_{2}, N_{2}}(\xi)\right|\right\|\right|^{2} /\left\|\left|X_{1, N_{2}}(\xi)\right|\right\|^{2}$ is constructed for $N_{2}=1800$ and is shown in Fig. 5. The norm \|\|$\cdot \|$ is such that $\|\mid \cdot\| \|^{2}=\left\langle\left.\|\cdot\|\right|^{2}\right\rangle$ in which $\|$.$\| is the Euclidean norm. It can be seen that a reasonable convergence is reached$ for $P_{2}=4$. It should be noted that this convergence result does not depend on the value of $\ell$. blueThe required order $P_{2}$ for the solution depends simultaneously on the strength of uncertainty in the system description (i.e. in matrix $A(\omega)$ ) as well as on the structure of the dependence of the solution on the system randomness.

7.4.3. Convergence analysis of the stochastic preconditioner with respect to the number of samples. In order to study the convergence of the chaos decomposition of the stochastic preconditioner with respect to the numbers $N_{1}$ and $N_{1}^{\prime}$ of samples, the graphs of the mappings $N_{1} \mapsto\left\||| D_{P_{1}, N_{1}}^{\text {inc }}(\xi ; \ell) \mid\right\|^{2}$ and $N_{1}^{\prime} \mapsto\|\| L_{P_{1}^{\prime}, N_{1}^{\prime}}^{\text {inc }}(\xi ; \ell) \|\left.\right|^{2}$ are constructed for $P_{1}=2$ and $P_{1}^{\prime}=0$ and 
for different values of bandwidth $\ell$. These graphs are displayed in Fig. 6 and 7 . It can be seen that a reasonable convergence of $D_{P_{1}, N_{1}}^{\text {inc }}(\xi ; \ell)$ is reached for $N_{1}=4500$ while the convergence of $L_{P_{1}^{\prime}, N_{1}^{\prime}}^{\mathrm{inc}}$ is reached for $N_{1}^{\prime}=1$ (deterministic quantity).

It should be noted that the numerical cost of the incomplete factorization of matrix $A\left(\xi^{(r)}\right)$ given $L^{\mathrm{inc}}\left(\xi^{(r)} ; \ell\right)$ is smaller than the numerical cost of the incomplete factorization of matrix $A\left(\xi^{(r)}\right)$ for which $L^{\text {inc }}\left(\xi^{(r)} ; \ell\right)$ and $D^{\text {inc }}\left(\xi^{(r)} ; \ell\right)$ have to be computed. In the present case for which $L^{\text {inc }}\left(\xi^{(r)} ; \ell\right)=L^{\text {inc }}(\ell)$ is independent of $\left(\xi^{(r)}\right.$, this property results in a significant gain. Such a method can be used whenever $N_{1}^{\prime} \ll N_{1}$ (which is presently the case since $N_{1}=4500$ and $\left.N_{1}^{\prime}=1\right)$.

7.4.4. Convergence analysis of the stochastic solution with respect to the number of samples. For studying the convergence analysis of the chaos decomposition of $X_{P_{2}, N_{2}}(\xi)$ with respect to the number $N_{2}$ the graph of the mapping $N_{2} \mapsto\left\||| X_{P_{2}, N_{2}}(\xi) \mid\right\|^{2}$ is constructed for $P_{2}=4$ and is displayed in Fig. 8. It can be seen that the convergence is reached for $N_{2}=1800$. It should be noted that this convergence result does not depend on the value of $\ell$.

\subsection{Efficiency of the stochastic preconditioner}

In this section, we compare the numerical costs of the stochastic solution construction of Eq. (34) using the stochastic preconditioner and using the usual preconditioner for the following conditions: $N_{1}=4500, N_{1}^{\prime}=1, N_{2}=1800, P_{1}=2, P_{1}^{\prime}=0$ and $P_{2}=4$.

The numerical cost for the construction of the stochastic preconditioner is denoted by $C_{1}(\ell)$ and depends on its bandwidth $\ell$. The numerical cost for the construction of the stochastic solution using the stochastic preconditioner without including $C_{1}(\ell)$ is denoted by $C_{2}(\ell)$. Finally, the numerical cost for constructing the stochastic solution using the usual preconditioner is denoted by $C_{3}(\ell)$. It should be noted that the construction of the stochastic preconditioner is presented separately because its construction can generally be performed in an initial step. In addition, we present normalized numerical costs with respect to the total cost for the construction of the stochastic solution, that is to say, (1) the normalized numerical cost eff $1(\ell)=C_{1}(\ell) / C_{3}(\ell)$ for the construction of the stochastic preconditioner, and (2) the normalized numerical cost $\mathrm{eff}_{2}(\ell)=C_{2}(\ell) / C_{3}(\ell)$ for the construction of the stochastic solution using the stochastic preconditioner. Figure 9 displays the graph of function $\ell \mapsto$ eff $_{1}(\ell)$. It can be seen that, as expected, this normalized cost increases with the bandwidth. Figure 10 displays the graph of function $\ell \mapsto \operatorname{eff}_{2}(\ell)$ which shows that the gain increases with the bandwidth (that is also understandable). Comparing Figs. 9 and 10, it can be seen that the gain corresponds to the total cost of the usual preconditioner which is constructed inside the samples loop while the stochastic preconditioner is constructed in an initial step, outside the samples loop. It is clear from this argument that the proposed stochastic preconditioning is particularly suitable for problems in which the construction of the stochastic preconditioner can be performed outside all the main loops of the problem under consideration such as the time loop for evolution problems, and the equilibrium iterative loop for nonlinear problems. Moreover, it should be noted that, the greater the number of samples required in the MCS method, the greater the efficiency. 


\section{Conclusions}

The construction of a stochastic preconditioner using the chaos decomposition is proposed. The convergence properties of this stochastic preconditioner are studied through a numerical example which shows that a significant gain is obtained. In addition, for the treated example, it has been seen that, the convergence of the chaos decompositions of the diagonal and triangular parts of the stochastic preconditioner is reached for different values of the decomposition chaos order. This property allows the numerical effort associated with the construction of the stochastic preconditioner to be further decreased. The persistence of this very useful property merits further investigation in connection with a broader classes of random matrices.

\section{ACKNOWLEDGEMENT}

The financial support of the Office of Naval Research under grant number N00014-99-1-0900 is gratefully acknowledged.

\section{REFERENCES}

1. Barrett, R., Berry, M., Chan, T. F., et al. (1994), "Templates for the Solution of Linear Systems: Building Blocks for Iterative Methods", SIAM, Philadelphia.

2. Babuska, I. and Chatzipantelidis, P. (2002) "On solving elliptic stochastic partial differential equations," Computer Methods in Applied Mechanics and Engineering, Vol. 191, pp. 4093-4122.

3. Benzi, M. Meyer, C. and Tuma, M., "A sparse approximate inverse preconditioner for the conjugate gradient method," SIAM J. Sci. Comput., 17 (1996), pp. 11351149.

4. Ghanem, R., and Spanos, P. (1991) Stochastic Finite Elements: A Spectral Approach, Springer-Verlag, New York.

5. Ghanem, R., and Red-Horse, J. (1999) "Propagation of uncertainty in complex physical systems using a stochastic finite element approach," Physica D, Vol. 133, No. 1-4, pp. 137-144.

6. Pellissetti, M. and Ghanem, R. (2002) "Iterative solution of systems of linear equations arising in the context of the stochastic FEM," Journal of Advances in Engineering Software, 31 607-616.

7. Golub, G.H., and Van Loan C.F.(1989) Matrix Computation, Second Edition, The Jhon Hopkins University Press, Baltimore.

8. Saad, Y. (1996) "Iterative Methods for Sparse Linear Systems " Preconditioning Techniques PWS Publishing Company, Chapter 10.

9. Schueller. G., (2001) "Computational stochastic mechanics - recent advances," Computers E Structures, 79 (22-25): 2225-2234.

10. Soize, C., and Ghanem, R. (2003) "Physical Systems with Random Uncertainties: Chaos representations with arbitrary probability measure," submitted to SIAM Journal of Scientific Computing.

11. Soize, C., Maximum entropy approach for modeling random uncertainties in transient elastodynamics, $J$. Acoust. Soc. Am., 109(5), 1979-1996 (2001)

12. Soize, C., Random matrix theory and non-parametric model of random uncertainties, Journal of Sound and Vibration, 263, 893-916 (2003).

13. Wang, X., Gallivan, K.A., Bramley, R. (1994) "Incomplete Cholesky factorization with sparsity pattern modification", Technical Report 394, Indiana University-Bloomington, Bloomington, IN 47405.

14. Zhang, J., "A multilevel dual reordering strategy for robust incomplete LU factorization of indefinite matrices," SIAM J. Matrix Anal. Appl. 22 (3): pp. 925-947, 2000.

15. Zhang, Y., "Solving Large-Scale Linear Programs by Interior-Point Methods Under the MATLAB Environment", Department of Mathematics and Statistics, University of Maryland Baltimore County, Technical Report TR96-01. 


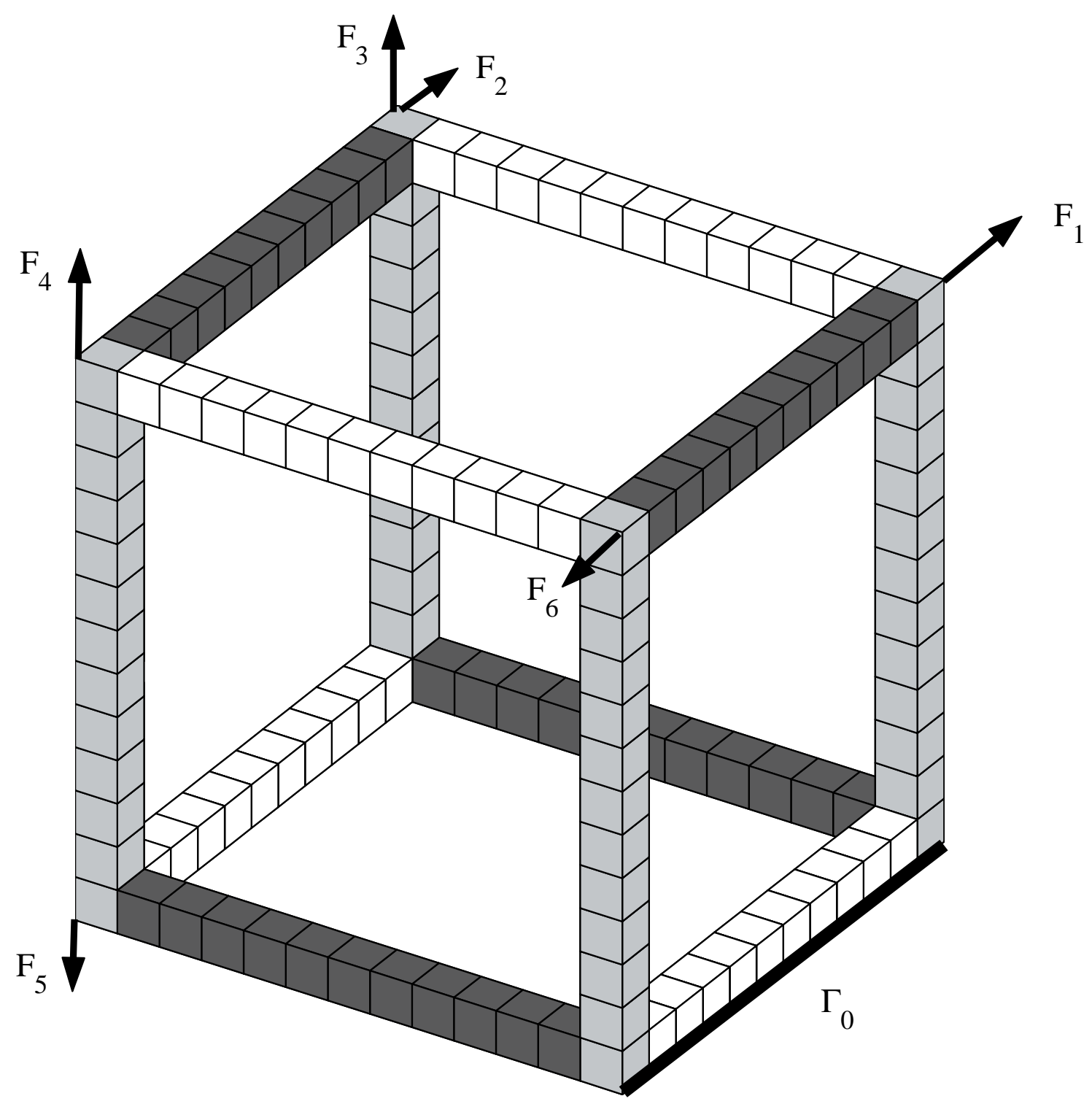

Figure 1. Geometrical definition and finite element mesh of the structural domain $\Omega$ and of the three subdomains $\Omega_{1}$ (gray), $\Omega_{2}$ (dark gray) and $\Omega_{3}$ (white). The external applied forces are indicated with arrows. Boundary $\Gamma_{0}$ is indicated by the thick dark solid line. 


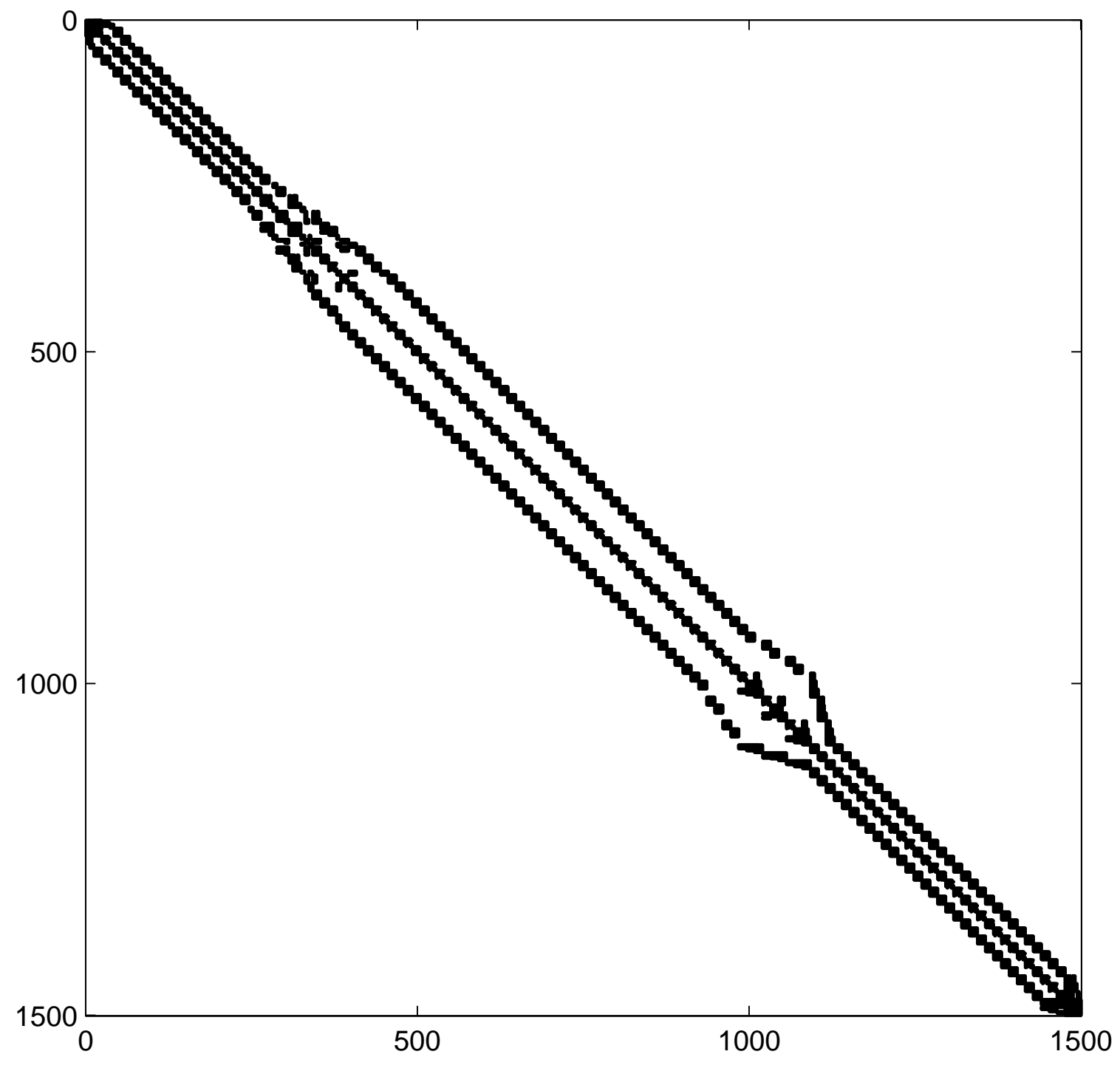

Figure 2. Sparsity pattern of matrix $A(\xi)$. 


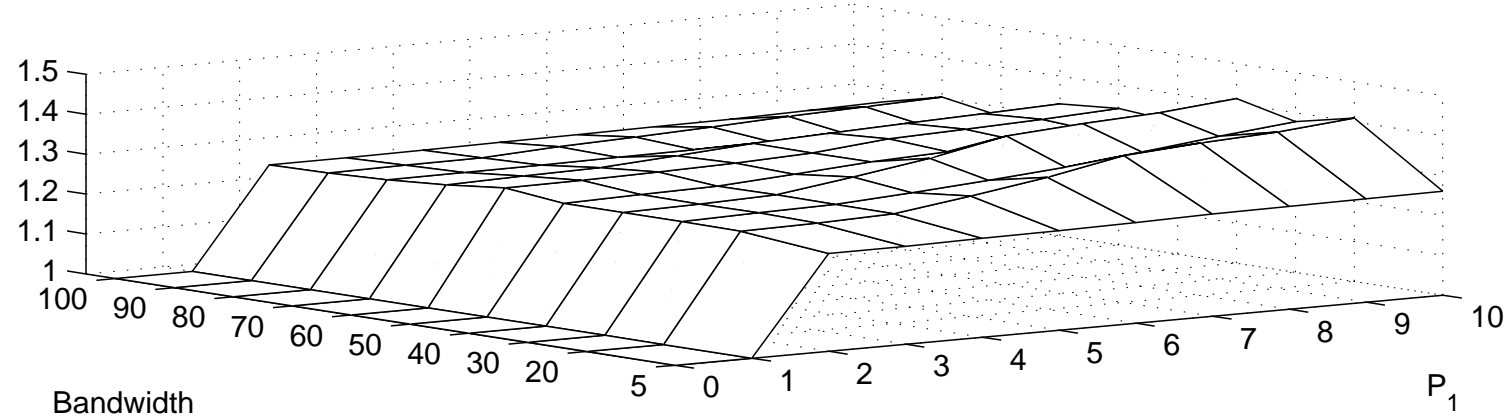

Figure 3. Convergence analysis of the chaos decomposition of $D^{\text {inc }}(\xi ; \ell)$ with respect to chaos decomposition order $P_{1}$ and bandwidth $\ell$ : graph of the mapping $\left(P_{1}, \ell\right) \mapsto \| D_{P_{1}, N_{1}}^{\text {inc }}$ $(\xi ; \ell)\left\|\left.\right|^{2} /\right\| D_{1, N_{1}}^{\text {inc }}(\xi ; \ell) \|\left.\right|^{2} ; N_{1}=8000$

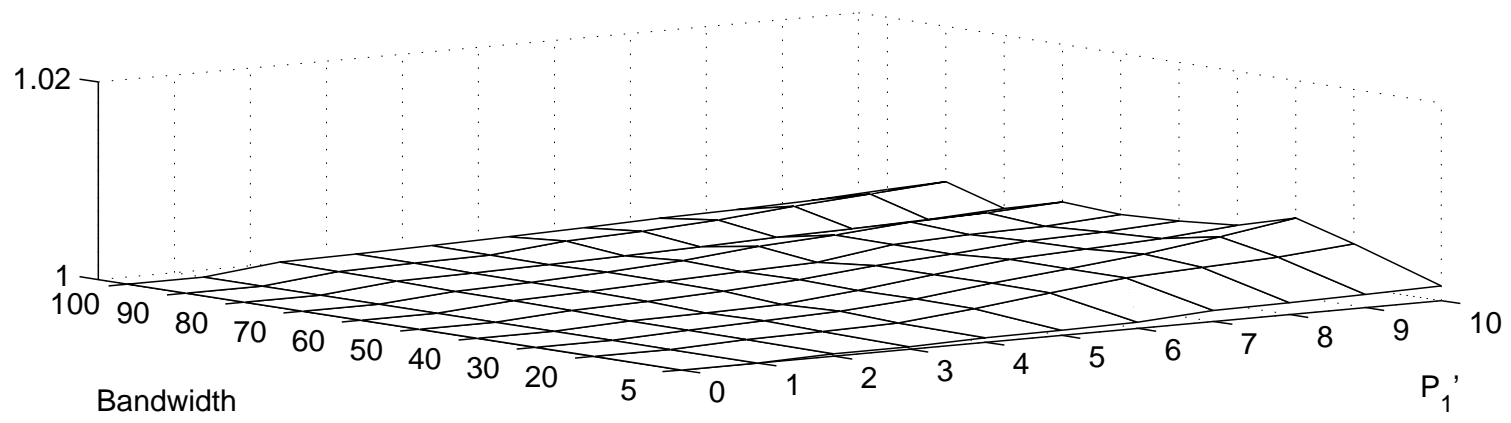

Figure 4. Convergence analysis of the chaos decomposition of $L^{\text {inc }}(\xi ; \ell)$ with respect to chaos decomposition order $P_{1}^{\prime}$ and bandwidth $\ell$ : graph of the mapping $\left(P_{1}^{\prime}, \ell\right) \mapsto\left\|L_{P_{1}^{\prime}, N_{1}^{\prime}}^{\text {inc }}(\xi ; \ell)\right\| \|^{2}$ $/\left\|L_{1, N_{1}^{\prime}}^{\text {inc }}(\xi ; \ell)\right\| \|^{2} ; N_{1}^{\prime}=8000$. 


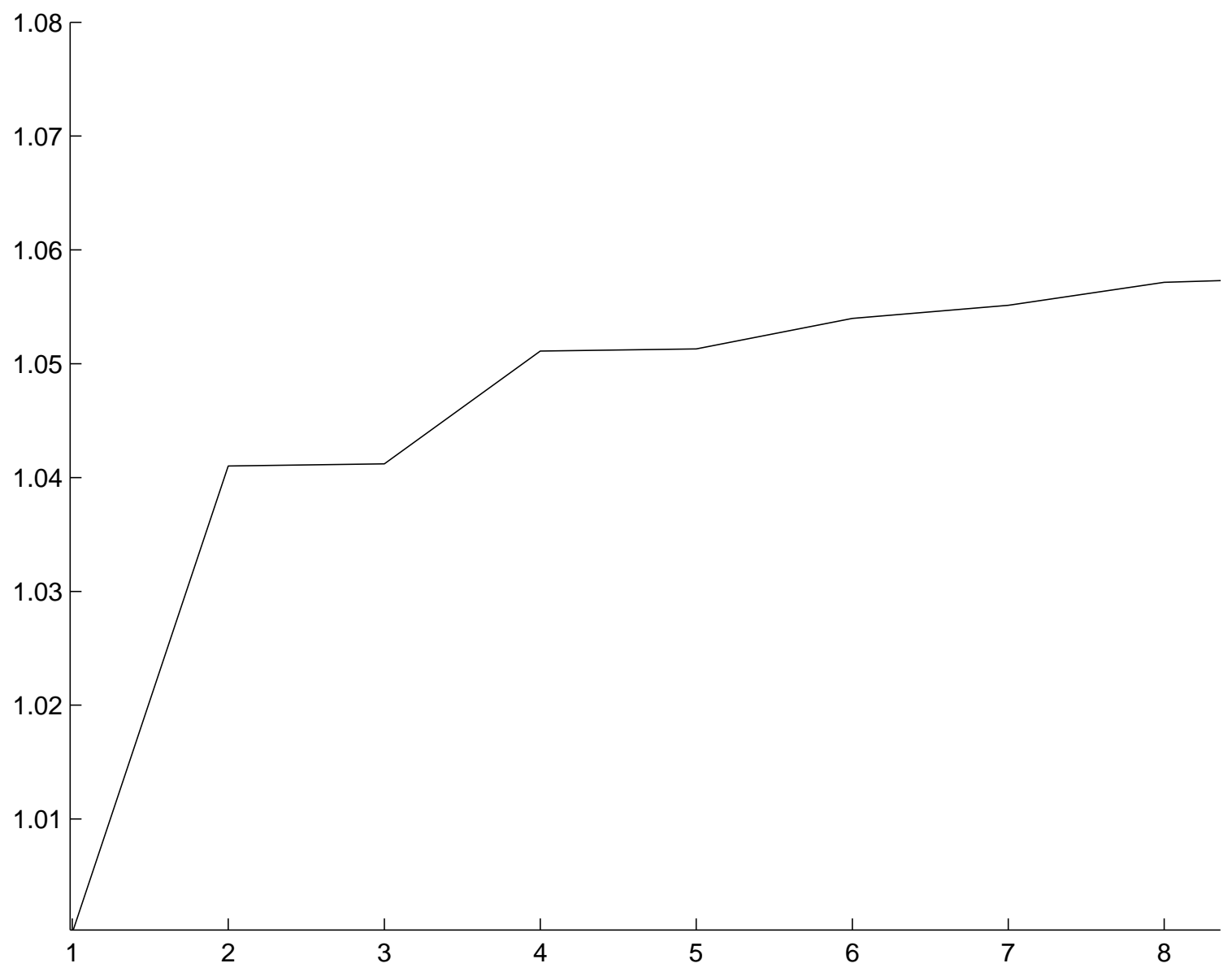

Figure 5. Convergence analysis of the chaos decomposition of $X_{P_{2}, N_{2}}(\xi)$ with respect to chaos decomposition order $P_{2}$ : graph of the mapping $P_{2} \mapsto\left\|\left.|| X_{P_{2}, N_{2}}(\xi)\left|\left\|\left.\right|^{2} /\right\|\right|\left|X_{1, N_{2}}(\xi)\right|\right|^{2} ; N_{2}=1,800\right.$. 


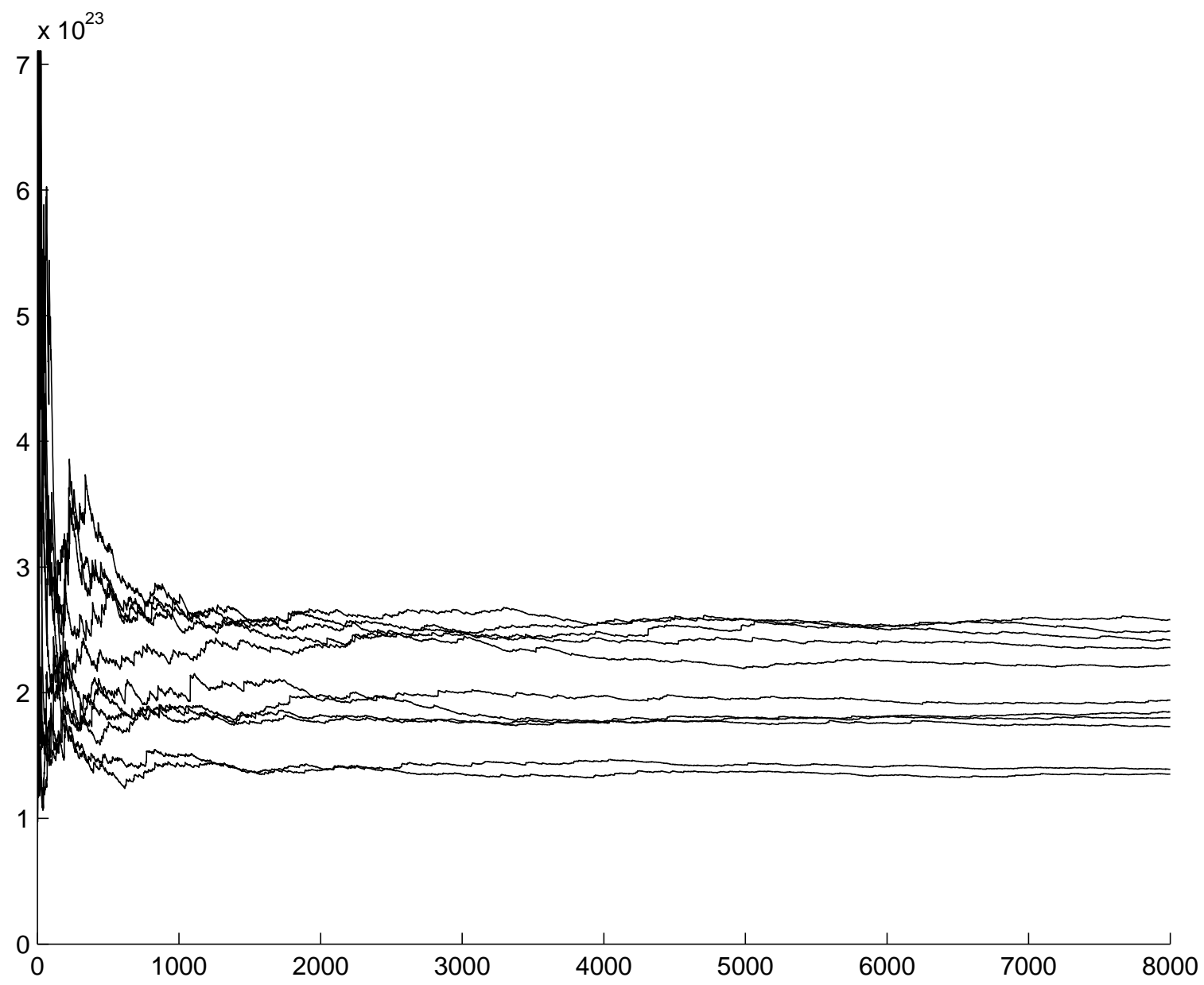

Figure 6. Convergence analysis of the chaos decomposition of $D_{P_{1}, N_{1}}^{\text {inc }}(\xi ; \ell)$ with respect to the number $N_{1}$ of samples for different values of banwidth $\ell$ : graphs of $N_{1} \mapsto\left\|\left|D_{P_{1}, N_{1}}^{\text {inc }}(\xi ; \ell)\right|\right\|^{2}$ for $P_{1}=2$ and for $\ell=5,10,20,30,40,50,60,70,80,90,100$. The norm decreases with increasing $\ell$. 


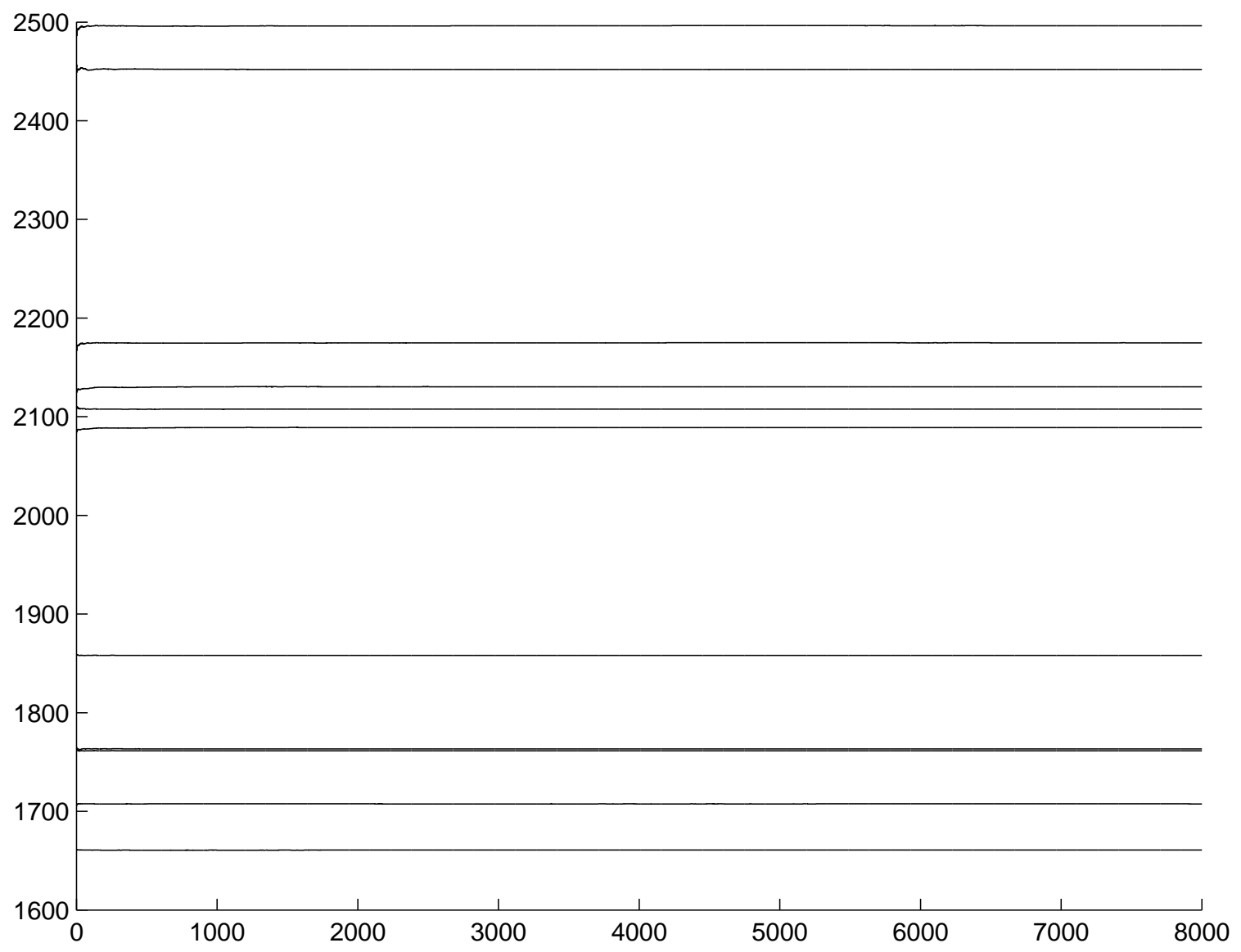

Figure 7. Convergence analysis of the chaos decomposition of $L_{P_{1}^{\prime}, N_{1}^{\prime}}^{\text {inc }}(\xi ; \ell)$ with respect to the number $N_{1}^{\prime}$ of samples for different values of banwidth $\ell$ : graphs of $N_{1}^{\prime} \mapsto\|\| L_{P_{1}^{\prime}, N_{1}^{\prime}}^{\text {inc }}(\xi ; \ell)\|\|^{2}$ for $P_{1}^{\prime}=0$ and for $\ell=5,10,20,30,40,50,60,70,80,90,100$. The norm decreases with increasing $\ell$. 


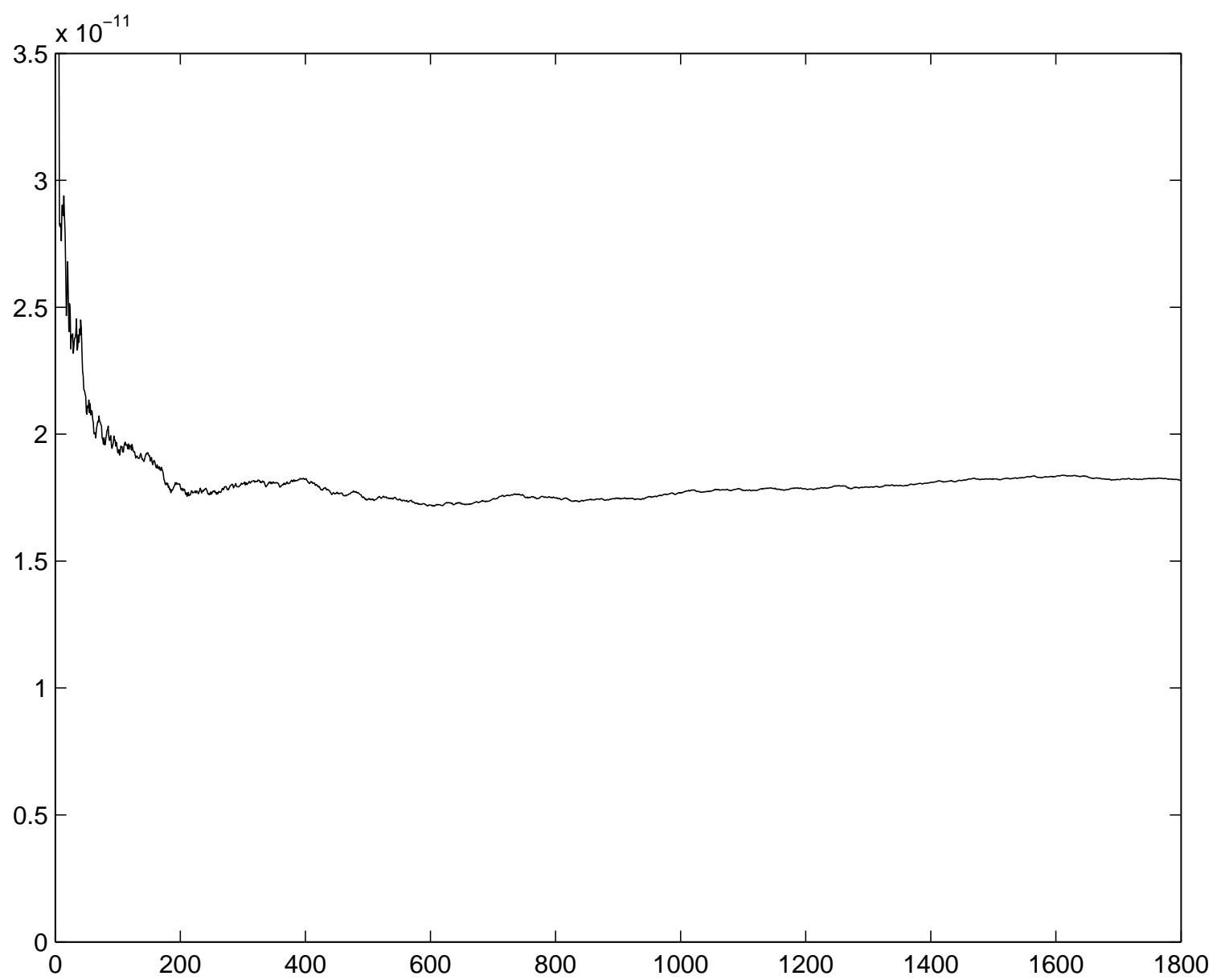

Figure 8. Convergence analysis of the chaos decomposition of $X_{P_{2}, N_{2}}(\xi)$ with respect to number $N_{2}$ of samples: graph of $N_{2} \mapsto\left\|\mid X_{P_{2}, N_{2}}(\xi)\right\| \|^{2}$ for $P_{2}=4$. 


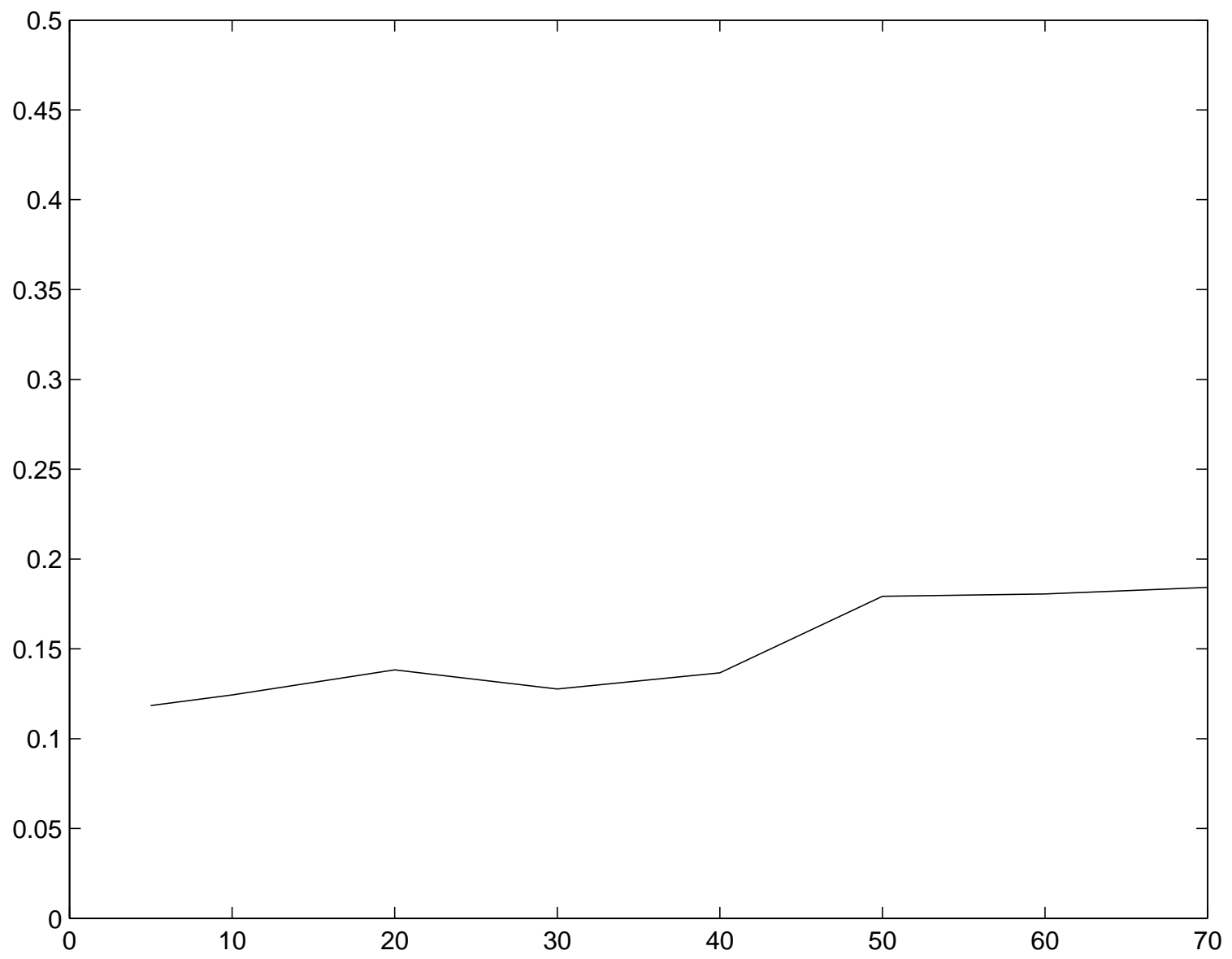

Figure 9. Normalized numerical cost for the construction of the stochastic preconditioner as a function of its bandwith $\ell$ : graph of $\ell \mapsto \operatorname{eff}_{1}(\ell) ; N_{1}=4,500, N_{1}^{\prime}=1 ; N_{2}=1,800 ; P_{1}=2, P_{1}^{\prime}=0 ; P_{2}=4$. 


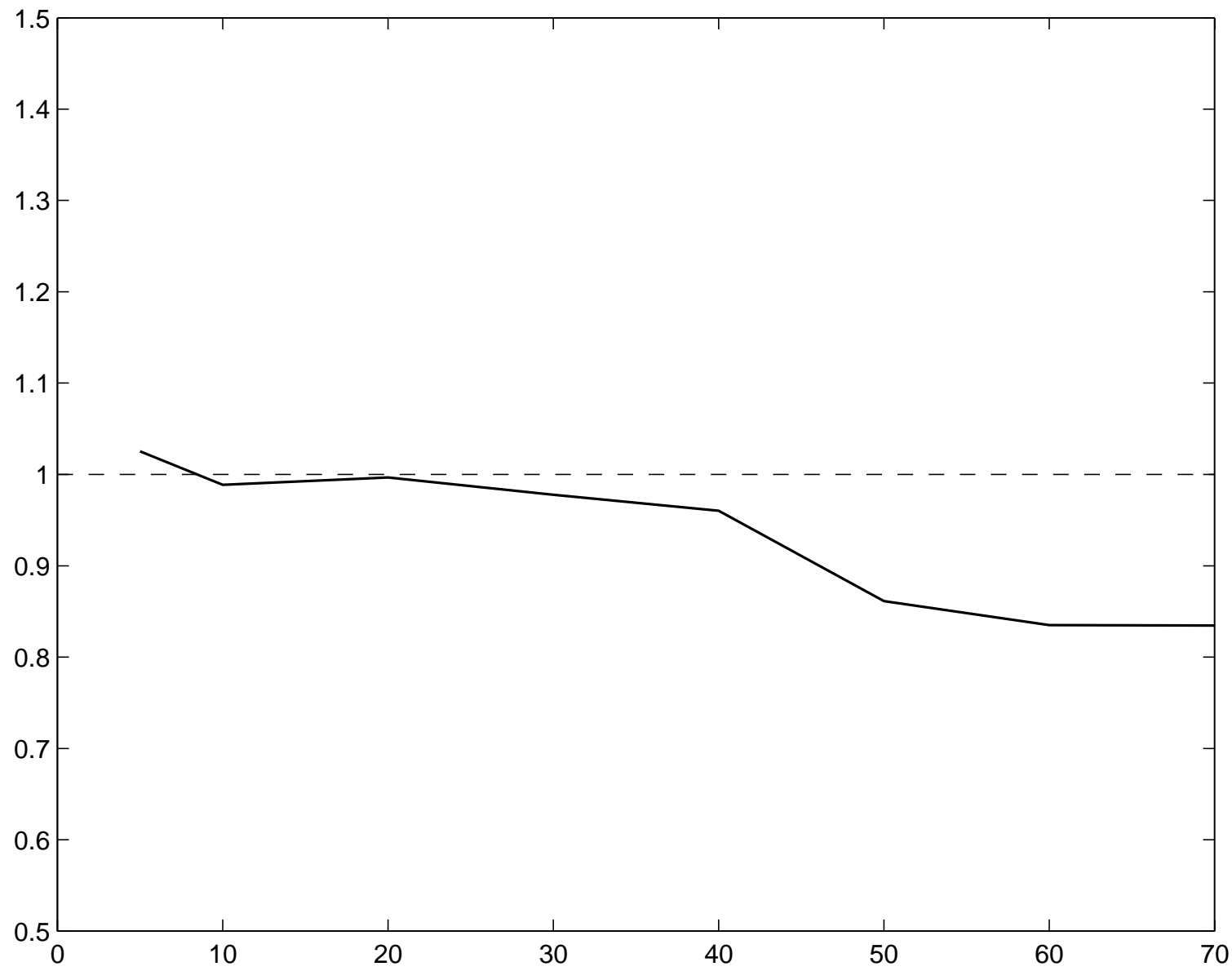

Figure 10. Efficiency analysis for the construction of the stochastic solution using the stochastic preconditioner as a function of its bandwidth $\ell$ : graph of $\ell \mapsto \operatorname{eff}_{2}(\ell)$ (solid line). The dashed line represents the normalized reference numerical cost corresponding to $C_{3}(\ell)$. 